\title{
Asymptotic Stability of Runge-Kutta Methods for Linear Delay-integro-diffrential-Algebraic Equation
}

\author{
Qiong Li and Yuxia Wang \\ School of Mathematics and Physics \\ Hubei Polytechnic University Huangshi \\ Hubei, China
}

\begin{abstract}
This paper deals with the asymptotic behavior of Runge-Kutta methods for linear delay-integro-differential-algebraic equations. It was shown that R-K of A-stability preserves the asymptotic stability of the equations under some conditions if applied to the equations.
\end{abstract}

Keywords-delay-differential-algebraic equations; Runge-Kutta methods; asymptotic stability

I. The Asymptotic StabiLity Of The Equations

We consider the delay integral differential algebraic system as follows:

$$
\left\{\begin{array}{c}
A x^{\prime}(t)+B x(t)+C x^{\prime}(t-\tau)+D x(t-\tau)+G \int_{t-\tau}^{t} x(s) d s=0, t \geq 0 \\
x(t)=\phi(t),-\tau \leq t \leq 0
\end{array}\right.
$$

It is assumed that the coefficient matrix A,B,C,D,G $\in R^{d \times d} \quad$ are all upper triangular matrix. $\phi(t) \in C^{d}$ is a d-dimensional initial vector which is known. The existence and uniqueness of solutions of system (1) is proven in the literature [1][2] as follows:

Theorem 1 If the coefficient matrices of the system (1) meet the following conditions:

For any

$$
\gamma \in R^{d},|<\gamma, A \gamma>| \geq|<\gamma, C \gamma>|
$$

If

$$
\begin{gathered}
\operatorname{det}[B+D+\tau G] \neq 0 \\
\operatorname{det}\left[s^{2} A+s B+G\right]=0, s \neq 0 \Rightarrow \operatorname{Re} s<0 \\
\sup _{\forall \operatorname{Re} s=0, s \neq 0} \rho\left[\left(s^{2} A+s B+G\right)^{-1}\left(s^{2} C+s D-G\right)\right]<1
\end{gathered}
$$

The system is asymptotically stable ${ }^{[3][4]}$. (In which $\rho[\cdot]$ indicates the matrix spectral radius.)

\section{ASYMPTOTIC STABILITY OF THE RUNGE-KUTTA METHOD}

The following Runge-Kutta method

$$
\begin{gathered}
y_{n+1}=y_{n}+\sum_{i=1}^{s} b_{i} f\left(t_{n}+c_{i} h, Y_{i}\right), \\
Y_{i}=y_{n}+h \sum_{j=1}^{s} a_{i j} f\left(t_{n}+c_{j} h, Y_{j}\right), i=1,2, \cdots, s
\end{gathered}
$$

was applied to the scalar test equation:

$$
y^{\prime}(t)=\lambda y, y\left(t_{0}\right)=y_{0}, \operatorname{Re} \lambda<0
$$

We can get the following conclusion:

$$
\begin{gathered}
y_{n+1}=y_{n}+h \lambda b^{T} Y \\
Y=e y_{n}+h \lambda A Y
\end{gathered}
$$

In which

$$
b=\left(b_{1}, b_{2}, \cdots, b_{s}\right)^{T}, Y=\left(Y_{1}, Y_{2}, \cdots, Y_{s}\right)^{T}, e=(1,1, \cdots, 1)^{T} \text {. }
$$

If the s-th order matrices $(I-h \lambda A)$ is nonsingular, we get

$$
y_{n+1}=\left[1+h \lambda b^{T}(I-h \lambda A)^{-1} e\right] y_{n}=R(z) y_{n}
$$

in which $z=h \lambda, R(z)=1+z b^{T}(I-z A)^{-1} e$, and $\mathrm{R}(\mathrm{z})$ is called the stable functions of the method.

Definition 1 If the method (6 7) is applied to the experimental equation, and the stability function satisfies the condition of $|\operatorname{Re}(z)|<1$, we say that the method is strictly A stable $^{[5]}$. $S_{R}:\{z: \operatorname{Re} z<0,|R(z)|<1\}$ is called absolute stability region of the method (6 7).

The s-th order R-K method $(\tilde{A}, \tilde{b}, \tilde{c})$ is applied to the experimental equation (1), in which

$$
\begin{aligned}
& \quad \tilde{A}=\left(\tilde{a}_{i j}\right)_{s \times s}, \tilde{b}=\left(\tilde{b}_{1}, \tilde{b}_{2}, \cdots, \tilde{b}_{s}\right)^{T}, 0<\tilde{c}_{i}<1, \text { and } \\
& \tilde{c}_{i}=\sum_{j-1}^{s} \tilde{a}_{i j}(i=1,2, \cdots, s) .
\end{aligned}
$$

The step is taken as $h=\tau / m$, $\mathrm{m}$ is any positive integer, the knot is taken as $t_{n}=t_{0}+n h$

$$
A X_{n i}^{\prime}+B\left(x_{n}+\sum_{j=1}^{s} \tilde{a}_{i j} h X_{n j}^{\prime}\right)+C X_{n-m i}^{\prime}+D\left(X_{n-m}+\sum_{j=1}^{s} \tilde{a}_{i j} h X_{n j}^{\prime}\right)+G \sum_{q=0}^{m} h v_{q}\left(X_{n-q}+\sum_{j=1}^{s} \tilde{a}_{i j} h X_{n-q i}^{\prime}\right)=O
$$




$$
x_{n+1}=x_{n}+\sum_{i=1}^{s} \tilde{b_{i}} h X_{n i}^{\prime}
$$

In which

$$
\begin{gathered}
X_{n i}^{\prime} \approx x^{\prime}\left(t_{n}+c_{i} h\right) \\
x_{n}+\sum_{j=1}^{s} \tilde{a}_{i j} h X_{n j}^{\prime} \approx x\left(t_{n}+c_{i} h\right) \\
h \sum_{q=0}^{m} v_{q}\left(x_{n-q}+\sum_{j=1}^{s} \tilde{a}_{i j} h X_{n-q j}^{\prime}\right) \approx \int_{t_{n}+c h=\tau}^{t_{n}+c h_{i}} x(s) d s
\end{gathered}
$$

The coefficient $v_{q}(q=0,1, \cdots, m)$ is determined by the following compound integral formula in which the order of convergence is $p=2 s$.

$$
\int_{0}^{\tau} x(s) d s=h \sum_{q=0}^{m} v_{q} x((m-q) h)+O\left(h^{2 s+1}\right)
$$

We denote

$$
\begin{gathered}
K_{n i}=h X_{n i}^{\prime} \in C^{d}, i=1,2, \cdots, d \\
K_{n}=\left[K_{n 2}^{1}, K_{n 2}^{1}, \cdots, K_{n s}^{1}, K_{n 1}^{2}, \cdots, K_{n s}^{2} \cdots, K_{n 1}^{d}, \cdots, K_{n s}^{d}\right] \in C^{d \times s}
\end{gathered}
$$

So the equations (8)(9)can be written as

$$
\begin{aligned}
& {\left[\begin{array}{cc}
A \otimes I_{s}+h\left(B+v_{0} h G\right) \otimes \tilde{A} & O \\
-I_{d} \otimes \tilde{b}^{T} & I_{d}
\end{array}\right]\left[\begin{array}{c}
K_{n} \\
x_{n+1}
\end{array}\right]} \\
& +\left[\begin{array}{cc}
v_{1} h^{2} G \otimes \tilde{A} & h\left(B+v_{0} G\right) \otimes e \\
O & I_{d}
\end{array}\right]\left[\begin{array}{c}
K_{n-2} \\
x_{n-1}
\end{array}\right] \\
& +\left[\begin{array}{cc}
v_{2} h^{2} G \otimes \tilde{A} & h^{2} v_{1} G \otimes e \\
O & O
\end{array}\right]\left[\begin{array}{c}
K_{n-2} \\
x_{n-1}
\end{array}\right]+\cdots+ \\
& +\left[\begin{array}{cc}
v_{m-1} h^{2} G \otimes \tilde{A} & h^{2} v_{m-2} G \otimes e \\
O & O
\end{array}\right]\left[\begin{array}{l}
K_{n-m+1} \\
x_{n-m+2}
\end{array}\right] \\
& +\left[\begin{array}{cc}
C \otimes I_{s}+h\left(D+v_{m} h G\right) \otimes \tilde{A} & h^{2} v_{m-1} G \otimes e \\
O & O
\end{array}\right]\left[\begin{array}{c}
K_{n-m} \\
x_{n-m+1}
\end{array}\right] \\
& +\left[\begin{array}{cc}
O & h\left(D+v_{m} h G\right) \otimes e \\
O & O
\end{array}\right]\left[\begin{array}{c}
K_{n-m-1} \\
x_{n-m}
\end{array}\right]=\mathrm{O}
\end{aligned}
$$

The corresponding characteristic equation is: $p(z)=\operatorname{det}\left[\begin{array}{ll}T_{11} & T_{12} \\ T_{21} & T_{22}\end{array}\right]$ in which:

$T_{11}=z^{m+1}\left\{A \otimes I_{s}+h\left[B+\left(v_{0}+\frac{v_{1}}{Z}+\frac{V_{2}}{z^{2}}+\cdots+\frac{v_{m-1}}{z^{m-1}}\right) h G\right] \otimes \tilde{A}\right\}+z\left[C \otimes I_{s}+h\left(D+v_{m} h G\right) \otimes \tilde{A}\right]$

$T_{12}=z^{m}\left\{h\left[B+\left(v_{0}+\frac{v_{1}}{z}+\frac{v_{2}}{z^{2}}+\cdots+\frac{v_{m-1}}{z^{m-1}}\right) h G\right] \otimes e\right\}+h\left(D+v_{m} h G\right) \otimes \tilde{A}$

$$
T_{21}=-z^{m+1} I_{d} \otimes \tilde{b}^{T}, T_{22}=z^{m+1} I_{d}-z^{m} I_{d}
$$

We denote

$$
\bar{B}=B+\left(v_{0}+\frac{v_{1}}{Z}+\cdots+\frac{v_{m-1}}{Z^{m-1}}\right) h G
$$

$$
\bar{D}=D+v_{m} h G
$$

so we can get that

$$
\begin{gathered}
T_{11}=z^{m+1}\left(A \otimes I_{s}+h \bar{B} \otimes \tilde{A}\right)+z\left(C \otimes I_{s}+h \bar{D} \otimes \tilde{A}\right) \\
T_{12}=z^{m} h \bar{B} \otimes e+h \bar{D} \otimes \tilde{A}
\end{gathered}
$$

By the difference equation theory we know that If the characteristic equation satisfies the condition:

$$
p(z)=0 \Rightarrow|z|<1
$$

we have $x_{n} \rightarrow 0(n \rightarrow \infty)$, That is, the numerical method is asymptotically stable ${ }^{[5]}$. So we can Prove $p(z) \neq 0$ under certain conditions $|z \geq 1|$ as follows:

First we prove $T_{11}$ is a nonsingular matrix. By assuming we know $\bar{B}, \bar{D}$ are also upper triangular matrices, its main diagonal elements are set respectively as : $\bar{b}_{i}, \bar{d}_{i}(i=1,2, \cdots, d)$, then

$\operatorname{det} T_{11}=\prod_{i=1}^{d} \operatorname{det}\left[z^{m+1}\left(a_{i} I_{s}+h \bar{b}_{i} \tilde{A}\right)+z\left(c_{i} I_{s}+h \bar{d}_{i} \tilde{A}\right)\right]$

$\left.=\prod_{i=1}^{d} \operatorname{det}\left[z^{m+1}\left(a_{i}+c_{i} z^{-m}\right) I_{s}+h z^{m+1}\left(\bar{b}_{i}+\bar{d}_{i} z^{-m}\right) \tilde{A}\right)\right]$

$=\prod_{i=1}^{d} q_{i}(z)$

If $a_{i} \neq 0$,We suppose that $c_{i} \neq 0,\left|a_{i}\right|>\left|c_{i}\right|$ and $|z| \geq 1$, we can conclude that $\operatorname{Re} \frac{\bar{b}_{i}+\bar{d}_{i} z^{-m}}{a_{i}+c_{i} z^{-m}}>0$,

So $a_{i}+c_{i} z^{-m} \neq 0,(|z| \geq 1)$ and

$$
q_{i}(z)=\operatorname{det}\left[z^{m+1}\left(a_{i}+c_{i} z^{-m}\right)\left(I_{s}+h \frac{\bar{b}_{i}+\bar{d}_{i} z^{-m}}{a_{i}+c_{i} z^{-m}} \tilde{A}\right)\right.
$$

For all the eigenvalues of $\tilde{A}$ are real, we can get $q_{i}(z) \neq 0$; and some conclusions:

1. If $a_{i}=0$, we suppose $c_{i}=0$, if $|z| \geq 1$ and $\bar{b}_{i}+\bar{d}_{i} z^{-m} \neq 0$, we can conclude that $T_{11}$ is nonsingular:

$$
q_{i}(z)=\operatorname{det}\left[z^{m+1} h\left(\bar{b}_{i}+\bar{d}_{i} z^{-m}\right) \tilde{A}\right] \neq 0
$$

So we have

$$
\begin{aligned}
& p(z)=\operatorname{det}\left[\begin{array}{cc}
T_{11} & T_{12} \\
O & T_{22}-T_{21} T_{11}^{-1} T_{12}
\end{array}\right]=\operatorname{det}\left[T_{11}\right] \cdot \operatorname{det}\left[T_{22}-T_{21} T_{11}^{-1} T_{12}\right] \\
& \operatorname{det}\left[T_{22}-T_{21} T_{11}^{-1} T_{12}\right] \\
& =\prod_{i=1}^{d} z^{m}\left\{z-\left(1-\tilde{b}^{T}\left[\left(a_{i}+c_{i} z^{-m}\right) I_{s}+h\left(\bar{b}_{i}+\bar{d}_{i} z^{-m}\right) \tilde{A}\right]^{-1} h\left(\bar{b}_{i}+\bar{d}_{i} z^{-m}\right) e\right)\right\}
\end{aligned}
$$

For 
$1-\tilde{b}^{T}\left[\left(a_{i}+c_{i} z^{-m}\right) I_{s}+h\left(\bar{b}_{i}+\bar{d}_{i} z^{-m}\right) \tilde{A}\right]^{-1} h\left(\bar{b}_{i}+\bar{d}_{i} z^{-m}\right) e$

$=1-\tilde{b}^{T} \tilde{A}^{-1} e$, by the strict stability of Runge-Kutta method, we can get that: $\left|1-\tilde{b}^{T} \tilde{A}^{-1} e\right|<1$.

And on the condition of $|z| \geq 1$ and

$z^{m}\left\{z-\left(1-\tilde{b}^{T}\left[\left(a_{i}+c_{i} z^{-m}\right) I_{s}+h\left(\bar{b}_{i}+\bar{d}_{i} z^{-m}\right) \tilde{A}\right]^{-1} h\left(\bar{b}_{i}+\bar{d}_{i} z^{-m}\right) e\right)\right\} \neq 0$

we also can get that $\operatorname{det}\left[T_{11}\right] \operatorname{det}\left[T_{22}-T_{21} T_{11}^{-1} T_{12}\right] \neq 0$, and $p(z) \neq 0,(|z| \geq 1)$

2. If $a_{i} \neq 0, \quad c_{i} \neq 0$,We can get $a_{i}+c_{i} z^{-m} \neq 0,(|z| \geq 1)$ and

$1-\tilde{b}^{T}\left[\left(a_{i}+c_{i} z^{-m}\right) I_{s}+h\left(\bar{b}_{i}+\bar{d}_{i} z^{-m}\right) \tilde{A}\right]^{-1} h\left(\bar{b}_{i}+\bar{d}_{i} z^{-m}\right) e=1-R\left(-h \frac{\bar{b}_{i}+\bar{d}_{i} z^{-m}}{a_{i}+c_{i} z^{-m}}\right)$ $=\tilde{b}^{T}\left(I_{s}+h \frac{\bar{b}_{i}+\bar{d}_{i} z^{-m}}{a_{i}+c_{i} z^{-m}} \tilde{A}\right)^{-1} h \frac{\bar{b}_{i}+\bar{d}_{i} z^{-m}}{a_{i}+c_{i} z^{-m}} e$

In which $R(\hat{z})=1-\hat{z} \tilde{b}^{T}\left(I_{s}-\hat{z} \tilde{A}\right)^{-1} e$ is the stable functions of the strictly A stable Runge-Kutta method.

If $-h \frac{\bar{b}_{i}+\bar{d}_{i} z^{-m}}{a_{i}+c_{i} z^{-m}} \in S_{R}$ is satisfied, in which $S_{R}$ is stability range of the method, we can get that: on the condition of $|z| \geq 1$, the formula

$z^{m}\left\{z-\left(1-\tilde{b}^{T}\left[\left(a_{i}+c_{i} z^{-m}\right) I_{s}+h\left(\bar{b}_{i}+\bar{d}_{i} z^{-m}\right) \tilde{A}\right]^{-1} h\left(\bar{b}_{i}+\bar{d}_{i} z^{-m}\right) e\right)\right\} \neq 0$ is established.

\section{CONCLUSION}

Theorem 2 If the coefficient matrix of the system (1) and the chosen parameter $v_{q}(q=0,1, \cdots, m)$ of the integral formula satisfy:

(1) For any $\gamma \in R^{d},|<\gamma, A \gamma>| \geq|<\gamma, C \gamma>|$ is established.

(2)If $a_{i}=0$, we have $c_{i}=0(i=1,2 \cdots, d)$.

(3)If $a_{i} \neq 0$, we have $c_{i} \neq 0$ and $\left|a_{i}\right|>\left|c_{i}\right|$,

$$
(i=1,2, \cdots, d)
$$

(4)If $|z| \geq 1$, and $\operatorname{Re} \frac{\bar{b}_{i}+\bar{d}_{i} z^{-m}}{a_{i}+c_{i} z^{-m}}>0$

in which

$$
\bar{b}_{i}=b_{i}+\left(v_{0}+\frac{v_{1}}{z}+\cdots+\frac{v_{m-1}}{z^{m-1}}\right) h g_{i}
$$

$$
\bar{d}_{i}=d_{i}+v_{m} h g_{i}, \quad i=1,2, \cdots, d
$$

We get the conclusion: If $-h \frac{\bar{b}_{i}+\bar{d}_{i} z^{-m}}{a_{i}+c_{i} z^{-m}} \in S_{R}$ is satisfied, in which $S_{R}$ is the stability range of the method, The numerical solution of the A strict stable Runge - Kutta method $(\tilde{A}, \tilde{b}, c)$ is asymptotically stable, in which $\tilde{A}$ satisfies that the characteristic values of $\tilde{A}$ are all real.

\section{REFERENCES}

[1] J K Hale, S M Verduyn Lunel. Introduction to functional differential equations.[M]. Spring .Berlin, .1983

[2] U.Ascher, L.R.Retzold. The numerical solution of delay-differential-algebraic equations of retarded and neutral type.[J]. SIAM J.Numer.Anal.1995.32:1635-1657

[3] Zhu W, Petzold L.R. Asymptotic stability of linear delay differential-algebraic equations and numerical methods.[J]. J.Appl.Numer.Math.1997.24:247-264

[4] J J.Zhao, Y Xu, M Z Liu. Stability analysis of numerical methods for linear neutral Volterra delay-integro-differential system.[J].Applied Math and Computation.2005.167:1062-1069

[5] John F. Monahan. Numerical Methods of Statistics.[M].Cambridge University Press,2012:73 